\title{
Adjustment and Analysis of Time Delay in Active Control of Structure Based on Artificial Neural Network
}

\author{
Li Gang \\ The School of Computer Science and Technology of Huaiyin Normal University Jiangsu, Huaiyin, 223001
}

Keywords: artificial neural network; active control of structure; adjustment and analysis of time delay; research

\begin{abstract}
The development of ears is accompanied by the continuous innovation and development of science and technology, especially the development of biotechnology, which greatly improves people's understanding of biotechnology. Artificial neural network is a physical mechanism to simulate the information processing mechanism of human brain. The precursors of artificial intelligence are in preparation. The research on artificial neural network in China is relatively late, but it has made great contributions to the application of artificial neural network. Civil engineering experts have received much attention in recent years. Artificial neural network is suitable for structural analysis, design, detection and structural control. Based on artificial neural network that is commonly used in the development and structure of the control network model, artificial neural network is mainly studied in the time adjustment and analysis in the control of the organization, and its application foreground is prospected.
\end{abstract}

\section{Introduction}

Artificial neural network control is based on human neural network to construct sensible working mechanism, realize information data processing, learning, memory ability and thinking. Artificial neural network control is to use a neural network in the control system to model problems that abstraction is difficult to describe accurately, to give a more accurate analysis of the problem structure model. The time-adjustment and analysis of the artificial neural network in the active control of the structure is a good application prospect of civil engineering experts based on active control in the control of the architectural structure, but the measurement, signal transmission and calculation of the structure need to consume certain practices. Therefore, the control force is always later than the structural response. The late existence will lead to the law of active control. In order to eliminate this lag that does not contribute to control, civil engineering experts have applied the artificial neural network to the structure since the beginning of the new century. The time before the structure predicts the state to occur, thus providing a time difference that can be waited for active control, and then adjusting the effect of lateness.

\section{Application of Artificial Neural Network in Civil Engineering Structures}

\subsection{Structural modeling}

In civil engineering structure, is the most important application of modeling, because the wind load and earthquake excitation is random, so we must first construct feedback control method to control the response of structures, so we need the data modeling. General mathematical modeling and the level of the variable structure variables, and the structure of all kinds of uncertainty, it is too complicated to construct effective mathematical model, which is unfavorable to the design and control, so the lack of the structural calculation and the structure of the control model, the influence of it is difficult to control the quality of the impact of changing the structural part of the structure control. Artificial neural network has strong learning ability, and the control and response of neural network modeling and control algorithm is more perfect. It is another entry point for the development of structural control ${ }^{[1]}$. 


\subsection{Nonlinear control}

The control method of nonlinear control is for the intensity and stiffness degradation that appears in the actual structure, so the actual structural model correction is an important issue in the structural control. Using classical and modern control theory, it is sometimes difficult to achieve a feasible control system analysis and design. In the state space model, it is sometimes difficult to find the proper parameters for the nonlinear autoregressive moving average model, so the estimation result is not reliable. The theory and application of traditional nonlinear control methods are very flawed and difficult to be applied to the actual structure of the model components and control. Artificial neural networks can learn how to obtain the best approximation of nonlinear mapping capabilities, combined with the identification of nonlinear system models. And control, there is no restriction between nonlinear models, and it has strong advantages ${ }^{[2]}$.

\subsection{Late adjustments and analysis}

Time delay is the proper time difference between active control and structural response, which will affect the normal operation of the system. This is an important obstacle that researchers are trying to address. In operation control, the time and scale prediction of the next wave of earthquakes are rarely analyzed based on the traditional theory. Due to the serious non-linearity of the system, it is difficult to predict the ideal structure accurately, so the experts have to jump out of the traditional theoretical foundation. The development of modern technology. Artificial neural network can learn the system input and output through the square error and standard, therefore, artificial neural network and the system input and output neural network can be achieved within a relationship, was hidden in does not affect the output of neural network and system. The output is in error range. The characteristics of the system is that the neural network can obtain a reasonable equipped with adequate training sample data of neural network system, can accurately predict the next seismic wave, etc., and can predict the structure appeared a few minutes before. This enables active control to react in advance to achieve a consistent response to the structure. So as to eliminate the delay on the normal operation of the system.

\section{Application Condition of Time-delay Adjustment and Analysis of Artificial Neural Network in Active Structural Control}

\subsection{Foreign application condition}

Neural networks was first applied in controlin the 1960s, using a simple neural controller to control the movement of an inverted pendulum. In 1994 YEN realized a long-span spatial structure and structural control and recognition simulation of advanced space technologyusing a BP network and a radial basis function RBF neural network. In 1995, Ghaboussi developed a neural network controller that can realize the response of the three-story frame structure to earthquakes and the calculation of the single simulation control, using BP algorithm to complete the control algorithm, and the sensor to respond to the measurement mechanism, making the neural network controller consistent with the structure. Mortimer et al. used random search for global optimization to improve the neural network learning method, accelerated the learning speed and accuracy of the artificial neural network, extended the artificial neural network, realized the algorithm application of the multi-layer network, and verified the effect of neural network control with the random loads. The back-propagation efficient neural network controller developed by H.M.Chen in 1996 can achieve active structural control under dynamic loading, consisting of two parts, a neural simulation network and a nerve action network. It has been assessed effective using three earthquakes at the Morgan Building. Jaghatai and Ghaboussi then compared the three-tiered frame model's control effects of neural controllers, neuro-fuzzy controllers, and predictive optimization controllers under earthquakes. It was found that the neural controller can still have good stability under the damage of the mechanism, but its control effect is less than that of the neuro-fuzzy controller, predictive optimization controllers' control effect on the damage structure. The neuro-fuzzy controller is effective in the structure without damage or slight damage, and a good control effect can be 
obtained with a smaller control force ${ }^{[3]}$.

\subsection{Domestic applications situation}

The outstanding scholars intime-delay adjustment and analysis of artificial neural network in active structural control are Li Hongnan, Zhang Shunbao, Ji Dongwu, He Yuxi, etc. Each has his own team to study the neural network's intelligent control in the structure. Li Hongnan et al. explored the neural network intelligent control and prevention from the damping of the tuned liquid columnar damper of building structures, using the neural network model of the MR damper constructed with neural network technology to simulate the system characteristics, and then establishing the control system design adapted to the structural response. According to the effective analysis method, the structural control strategy is effectively discussed through digital simulation results ${ }^{[4]}$.

Zhang Shunbao, based on the BP network, specifically studied the time-delay adjustment of the neural network to the active structural control, selecting a reasonable amount of neural network input and hidden layer units, using the superior learning ability of the neural network to enhance certain Mapping capabilities, and then predicting future structural behavior based on the past data and reaction of the structure, which can buy waiting time for the active structural control of the signal measurement, transmission and the computer and servomechanism force process so as to adjust or eliminate the time delay of active structural control. This method was first applied in the real-time wind vibration prediction control of Nanjing TV Tower. The impact of wind vibration acceleration on the human body under the action of pulsating wind load of Nanjing TV station was used to enhance wind vibration control. The AMD control system first adopts a three-layer BP neural network to construct the organizational state prediction period. In order to improve the prediction accuracy, a three-layer BP neural network is constructed for each element, which can rapidly respond to the displacement and velocity of the particles, thereby improving the prediction structure and realize the time-delay adjustment of active control ${ }^{[5]}$.

Yan Dongwu mainly deals with structural response and control signal delay compensation, discusses predictive control algorithms based on multi-layer feed forward networks, and then implements vibration response control for multi-layer structures, enabling neural networks to maintain strong compensation under time delays to ensure the response speed and accuracy of the active control.

\section{Conclusion}

In summary, China is a late starterin time-delay adjustment and analysis of artificial neural network in active structural control. But at present there are also certain achievements and brilliant application prospects. At this stage, artificial neural networks are often used in active structural control in China to improve the control effect, speed and accuracy.

\section{References}

[1] Fan Shi. Research on artificial neural network and fuzzy logic method for intelligent control of structural vibration [D]. Dalian University of Technology, 2015.111-113

[2] Zhao Lin. Research onsemi-active control of structural vibration and its practicality [D]. Tianjin University, 2013.82-87

[3] Chen Gonglian, Liu Wenfeng, Bai Zhiqiang. Application of Artificial Neural Network in Structure Control [J]. Industrial Construction, 2006(S1): 243-246.

[4] Huang Yongan. Theory and application of neural network in active structural control [D]. Northwestern Polytechnical University, 2014.25-26

[5] Civilization, Chu Minping, Deng Zichen. Research on fine calculation of the time-delay problem of Active Control Structure under Harmonic Excitation [J]. Chinese Journal of Applied Mechanics, 2013(02):107-110+164. 